\title{
EEG-BASED DECODING OF AUDITORY ATTENTION TO A TARGET INSTRUMENT IN POLYPHONIC MUSIC
}

\author{
Giorgia Cantisani, ${ }^{1 *}$ Slim Essid, ${ }^{1}$ Gaël Richard, ${ }^{1}$ \\ ${ }^{1}$ LTCI, Télécom Paris, Institut Polytechnique de Paris, 75013, Paris, France \\ firstname.lastname@telecom-paristech.fr
}

\begin{abstract}
Auditory attention decoding aims at determining which sound source a subject is "focusing on". In this work, we address the problem of EEG-based decoding of auditory attention to a target instrument in realistic polyphonic music. To this end, we exploit a stimulus reconstruction model which was proven to decode successfully the attention to speech in multi-speaker environments. To our knowledge, this model was never applied to musical stimuli for decoding attention. The task we consider here is quite complex as the stimuli used are polyphonic, including duets and trios, and are reproduced using loudspeakers instead of headphones. We consider the decoding of three different audio representations and investigate the influence on the decoding performance of multiple variants of musical stimuli, such as the number and type of instruments in the mixture, the spatial rendering, the music genre and the melody/rhythmical pattern that is played. We obtain promising results, comparable to those obtained on speech data in previous works, and confirm that it is possible to correlate the human brain activity with musically relevant features of the attended source.
\end{abstract}

Index Terms - Auditory attention decoding, Polyphonic music, EEG, Stimulus reconstruction model

\section{INTRODUCTION}

Auditory attention is the cognitive mechanism that allows human beings to focus on a sound source of interest in every-day life environments. This allows the brain to extract and process high-level sound content effectively and efficiently. The target may be a sound source (a speaker, a siren, a musical instrument) and may carry spatial information (sound source localization, environment geometry, etc.). Auditory attention decoding aims at determining, from the brain's activity, which sound source a subject is "focusing on" while listening to a complex auditory scene.

Previous studies on speech attention decoding based on continuous magnetoencephalographic (MEG) [1-3] and electroencephalographic (EEG) signals [4-8] have shown that the neural activity tracks dynamic changes in the speech stimulus and can be successfully used to decode selective attention in a multispeaker environment. In all these works, a feature representation of the speech stimulus is reconstructed from the multi-channel EEG recordings through a stimulus reconstruction model, which is learned by solving a linear regression problem [8]. The authors of [6] were one of the first to show that such reconstructed feature representations (in this case spectrograms) were highly correlated with the salient

\footnotetext{
${ }^{*}$ This project has received funding from the European Unions Horizon 2020 research and innovation program under the Marie Skodowska-Curie grant agreement No. 765068
}

time-frequency features of the attended speaker's voice, and were only weakly correlated with the unattended speaker ones. When the stimulus consists of polyphonic music, one can recast this problem as that of decoding the attention to a particular musical instrument. However, this transposition is not straightforward since music represents a complex category of stimuli, entailing a variety of perceptual cues, linking to not only musical timbre, but also rhythm, harmony and melody.

A few attempts have thus been made at detecting and extracting music information from the brain's activity while the user is listening to realistic music, but none has addressed the question of attention decoding. Some studies aim at understanding how the brain processes basic structural components of music such as pitch [9-12], timbre [13-15] as well as sensory dissonance, highlevel melodic characteristics (e.g. melodic contour, key, mode, scale) and music-syntactic congruity [16]. The most studied components in the perception of music appear to be note onsets, beats, rhythm and meter [17-20]. Usually, the brain's reaction to such kind of structural components is studied using the event-related potential (ERP) paradigm, repeating the stimulus several times before averaging the corresponding EEG responses, in order to keep only the stimuli-relevant information and attenuate noise. This approach relies on specifically selected or designed short stimuli, which are appropriate to study only specific aspects of music (e.g. note onsets) or the reaction to isolated sounds. A different approach is needed to study the continuous brain response to a long and complex naturalistic stimulus such as a musical piece in its entirety. Only a few attempts have been made to track the dynamics of naturalistic music stimuli in the EEG signal. The authors of [17] found evidence that the time-domain audio envelope is consistently reflected in the EEG. In [21] the Mel spectrogram of both perceived and imagined naturalistic musical stimuli were reconstructed from the EEG while in [22] and [18], the same was done for the note onset sequence. However, these works were directly focusing on stimulus reconstruction and not on attended source decoding. Two attempts have been made for classifying the attended music [23] and the attended instrument [24] but both of them focused on the elicited ERPs.

In this context, we have studied the problem of decoding auditory attention to an instrument in realistic polyphonic music, i.e. recorded music, featuring two or three instruments played concurrently. For this purpose we exploit a stimulus reconstruction model successfully used for decoding attention to speech in multi-speaker environments [4-8], which, to our knowledge, was never previously applied to musical stimuli for attention decoding tasks. More particularly, we consider the challenging case of unaveraged EEG recordings (where the elicited responses are less visible) using polyphonic stimuli. It is worth noting that these stimuli were not specifically designed to elicit ERPs, and were played to the subjects using loudspeakers instead of headphones. We have investigated how the de- 

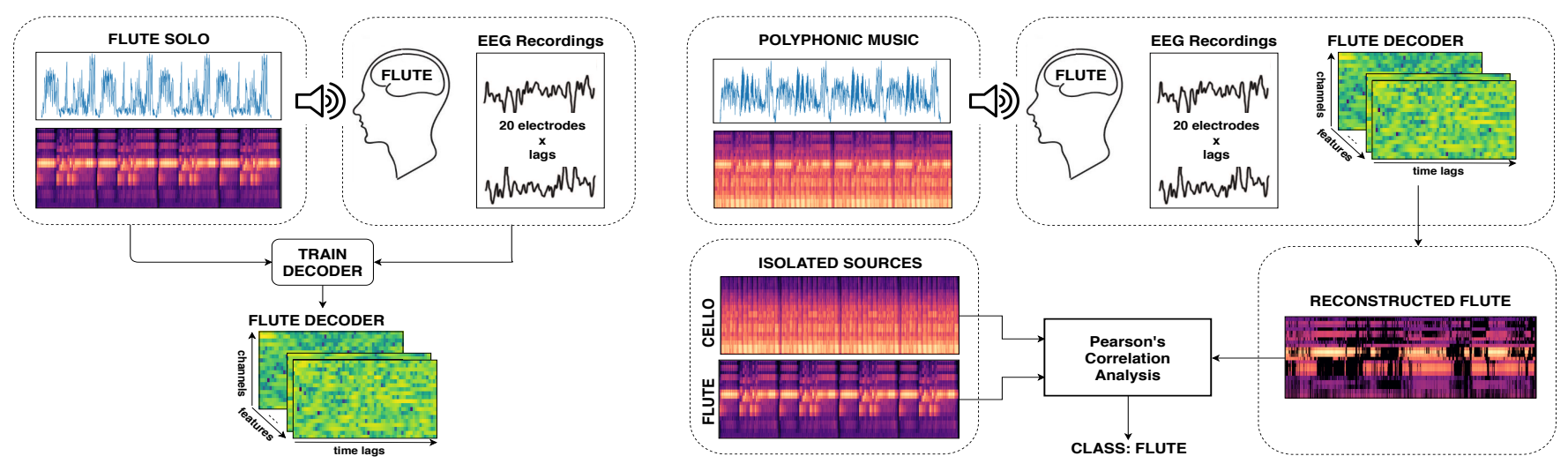

Figure 1: A subject-specific model is learned for each instrument from its solo and the EEG response collected while listening to it. Then, the same model is used to predict a representation of the attended instrument from the EEG response to a mixture containing that instrument. The attended instrument is the one which is mostly correlated with the reconstructed stimulus in terms of Pearson's correlation coefficient.

coding performance is influenced by the choice of audio representations as well as properties of the musical stimuli, such as number and type of instruments, spatial rendering, music genre, and the melody/rhythmical pattern that is played.

The remainder of the paper is organized as follows. The dataset is briefly discussed in Section 2. The audio stimuli representations, reconstruction and classification procedures are described in Section 3. Finally, the results are presented in Section 4 and future research directions are discussed in Section 5.

\section{DATA}

Studying the problem at hand requires data of well-synchronized musical stimuli and corresponding EEG responses which can only be acquired in a controlled sensory stimulation. There are a few publicly available music-related EEG datasets acquired in such a way [25-27], but they were designed for a different purpose and the subjects were not asked to attend to any particular instrument. The only one where participants were asked to focus on an instrument while listening to polyphonic music, is the music BCI dataset used in [24]. However, it was specifically designed for studying ERP-based attention decoding. Our focus is instead on single-trial attention decoding techniques, targeting real music compositions.

Consequently, we acquired our own dataset, named MADEEG $^{1}$, consisting of 20-channel surface EEG signals recorded from 8 subjects while they were attending to a particular instrument in polyphonic music, i.e. mixtures containing from two to three instruments. All subjects were non-professional/beginners musicians with different experience levels. Each subject listened to 78 stimuli, each one consisting of 4 repetitions of the same 6-second long music excerpt. This corresponds to approximately 30-32 minutes of 20-channel EEG recordings. Each attended instrument was previously heard in solo, as part of a training phase. The training excerpt was either from the same musical piece or another, and was played using either the same spatial rendering or a different one. Music stimuli were presented using two speakers positioned $\pm 45^{\circ}$ along the azimuth direction relative to the listener. The spatial rendering was implemented by using conventional stereo panning. The electrodes were placed according to the 10-20 international montage

\footnotetext{
${ }^{1}$ http://www.tsi.telecom-paristech.fr/aao/en/ 2019/07/19/mad-eeg/
}

system and were referenced to left mastoid in an unipolar setting. The sampling frequency was $256 \mathrm{~Hz}$. The acquired EEG data was synchronized with each stimulus, the $50 \mathrm{~Hz}$ power-line interference was removed using a notch filter and EOG/ECG artifacts were detected and removed using independent component analysis (ICA).

\section{METHODS}

The goal is to determine the attended instrument in a single-trial fashion based on 24-second long EEG excerpts aligned to corresponding audio stimuli (of the same length). Our approach can be summarized in two steps and is similar to the one commonly used for decoding the attention to speech [4-8]. It is shown in Figure 1. First we reconstruct an audio representation of the attended instrument from the single-trial EEG response of the user exploiting a decoder previously trained on solos of that instrument. Second, given the isolated instrumental tracks, the attended instrument is recognized as the one that has the highest correlation with the reconstructed stimulus in terms of Pearson's correlation coefficient.

\subsection{Audio Feature Extraction}

Three different audio representations were studied, one in the time domain and two in the time-frequency domain, in order to investigate which audio features are best suited to decoding the attention from the EEG. This choice can actually significantly impact the reconstruction quality and the decoding performance. In particular, we tested the time domain amplitude envelope computed using the Hilbert transform (AE) and the magnitude spectrogram (MAG) which have already shown good performance for speech stimulusreconstruction tasks [4-8]. A recent work explored auditory spectrograms modeling the peripheral auditory system [28] as they may better model how the attended source is represented in the EEG. We test instead the Mel spectrogram (MEL), a perceptually-scaled representation commonly used for music analysis.

\subsection{Stimulus Reconstruction}

The stimulus reconstruction model reconstructs an estimate of the stimulus representation $\hat{s}$ from multi-channel EEG data $r$ through a model $g$ which behaves like a multi-channel Wiener filter. This filter 
can be seen as a spatio-temporal decoder which linearly maps the neural activity back to the music representation, as a weighted sum of activity at each electrode in a given temporal context, as follows:

$$
\hat{s}(t, f)=\sum_{n} \sum_{\tau} g(\tau, f, n) r(t+\tau, n)
$$

where $\hat{s}$ is the predicted music representation at time $t$ and feature $f, r$ is the neural response of electrode $n$ at time $(t+\tau)$ and $g$ is the linear transformation function that depends on the time lag $\tau$, feature $f$, and electrode $n$. The time lag $\tau$ ranges between 0 and $\tau_{\max }$, i.e. the time interval where we assume to see the EEG response to the stimulus.

Equation (1) can be solved by minimizing the mean squared error between the actual and reconstructed stimulus representation, $\sum_{t} \sum_{f}[s(t, f)-\hat{s}(t, f)]^{2}$, which requires the calculation of the normalized reverse correlation: $\mathbf{G}(f)=\mathbf{C}_{\mathbf{R R}}^{-1} \mathbf{C}_{\mathbf{R S}}$, where $\mathbf{C}_{\mathbf{R R}}=$ $\mathbf{R R}^{T}$ is the auto-correlation of the EEG data and $\mathbf{C}_{\mathbf{R S}}=\mathbf{R S}^{T}$ is the cross-correlation of the stimulus and EEG data across all electrodes, time-lags for a given feature $f$. Thus the reconstruction of each feature $f$ of the stimulus representation $\hat{s}(t, f)$ is independent from the reconstruction of the other features and is obtained by convolving the relative multivariate impulse response $\mathbf{G}(f)$ with the EEG signal. In the case of magnitude and Mel spectrograms where the features correspond to frequency bins, the underlying assumption is that a spectrogram is a time-varying representation of the amplitude envelope at each frequency bin [5].

Since EEG signals are high-dimensional, autocorrelated, noisy data with high trial-to-trial variability, the estimate of the covariance matrices can be imprecise and subject to overfitting due to the high number of parameters to estimate [29]. Thus, a shrinkage regularization is used to constrain the model coefficients by smoothly penalizing extreme eigenvalues [29]: the diagonal of the autocovariance matrix $\mathbf{C}_{\mathbf{R R}}$ is then weighted as follows: $\mathbf{C}_{\mathbf{R R}}^{\prime}=$ $(1-\lambda) \mathbf{C}_{\mathbf{R} \mathbf{R}}+\lambda \nu \mathbf{I}$, where $\mathbf{I}$ is the identity matrix, $\nu$ is the average eigenvalue trace of $\mathbf{C}_{\mathbf{R} R}$, and $\lambda \in[0,1]$ is the smoothing parameter.

\section{EXPERIMENTAL RESULTS}

All audio representations were time aligned to the EEG responses acquired at $256 \mathrm{~Hz}$. Through a grid search over a set of reasonable values for each parameter $\left(\lambda \in[0.1,1], \tau_{\max } \in[250,500]\right.$ ms, number of Mel bands $\in[12,60])$, we found the best value for the shrinkage parameter to be $\lambda=0.1$, for $\tau_{\max }$ to be $250 \mathrm{~ms}$ post stimulus, and for the number of Mel bands to be 24, using the following train/test splits: for each of the 8 subjects train on 14 solos, test on 40 duets and 24 trios. The results are reported in Table 1 for different subsets of the test set corresponding to variants in the stimuli and different audio descriptors in terms of F1 scores. The considered variants consist of varying the number of instruments in the mixture, the melody that is played, the music genre and spatial rendering. Their statistical significance was assessed using an adaptation of the computationally-intensive randomization test [30], a non-parametric hypothesis test, comparing to chance, which does not make any assumption on the score distribution and can be used also for complex non-linear measures such as F1 score [31]. The considered significance levels are $5 \%, 1 \%$ and $0.1 \%$ and the tests were performed over $10^{4}$ iterations. Besides the decoding performance, we also evaluate the reconstruction capabilities of our system through the Pearson's correlation coefficient of the reconstructed stimulus representation with the attended in-

\begin{tabular}{|l|l|l|l|l|l|l|l|l|l|}
\hline \multirow{2}{*}{} & \multicolumn{9}{|c|}{ F1 score (\%) } \\
\cline { 2 - 10 } & \multirow{2}{*}{ all } & \multicolumn{2}{|c|}{ ensemble } & \multicolumn{2}{|c|}{ melody/rhythm } & \multicolumn{2}{c|}{ rendering } & \multicolumn{2}{c|}{ genre } \\
\cline { 3 - 10 } & & duets & trios & same & diff & mono & stereo & pop & classic \\
\hline AE & $51 *$ & $58 *$ & 37 n.s. & 48 n.s. & $53 *$ & $53 *$ & 48 n.s. & $54 *$ & 48 n.s. \\
\hline MAG & $72 * *$ & $74 * *$ & $66 * *$ & $76 * *$ & $\mathbf{6 5} * *$ & $73 * *$ & $\mathbf{7 2} * *$ & $\mathbf{6 4} * *$ & $79 * *$ \\
\hline MEL & $\mathbf{7 3} * *$ & $\mathbf{7 9} * *$ & $\mathbf{7 3} * *$ & $\mathbf{7 9} * *$ & $60 * *$ & $\mathbf{7 4} * *$ & $71 * *$ & $60 * *$ & $\mathbf{8 3} * *$ \\
\hline
\end{tabular}

Table 1: F1 scores for different subsets of the test set: all for all the test mixtures, duets and trios for those containing respectively 2 or 3 instruments, $s m$ and $d m$ for those which exhibit respectively the same or a different melody/rhythmical pattern as the solo used to train the model, mono and stereo for those rendered respectively in mono or stereo." “**” denotes high $(p<0.001)$, “*” good $(p<$ $0.01)$, and "n.s." no $(p>0.05)$ statistical significance of the results.

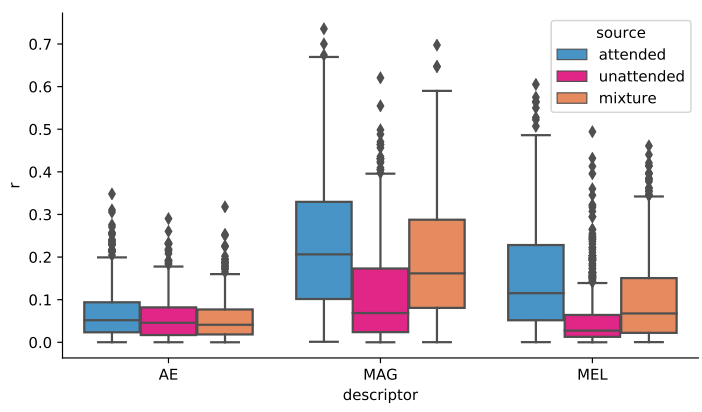

Figure 2: Pearson's correlation coefficients of the reconstructed stimulus with the attended source (blue), the unattended one (pink) and the mixture (orange) for the three audio descriptors.

strument $r_{\text {attended }}$, the unattended instrument $r_{\text {unattended }}$ and the mixture $r_{\text {mixture }}$.

In all cases except for the amplitude envelope, the F1 scores are statistically above chance $(p<0.001$ on a randomization test over 10000 repetitions). In fact, the decoding performance of this feature and its correlation scores are drastically lower than the one found for the two time-frequency descriptors (see Table 1 and Figure 2). Thus, the decoding seems to clearly benefit from the use of a finer audio representation, highlighting amplitude modulations in different frequency bands. Nevertheless, the $r_{\text {attended }}$ are comparable to the ones obtained in [7] for speech with the same audio descriptor (median $r=0.054$ ). However, our $r_{\text {attended }}$ values are close to $r_{\text {unattended }}$ and this led to a much lower decoding accuracy than [7]. One reason may be that the music envelopes are significantly different from the speech ones, as the complexity and modulations of music signals are higher, which makes it difficult to discriminate between the envelopes of the attended and the unattended instruments. Here the model is likely to account for effects more related to the whole mixtures than individual instruments, causing $r_{\text {attended }}$ and $r_{\text {mixture }}$ to be really similar. Moreover, we have to consider that in our case the stimuli were polyphonic and rendered through loudspeakers while in [7] two concurrent speech stimuli were presented as monaural sources using headphones playing a different source to each ear. In general, when both $r_{\text {attended }}$ and $r_{\text {unattended }}$ are low and similar, it means that the quality of the reconstructed stimulus is bad, hence it is difficult to decode the attended instrument. Since the performance of the AE are drastically lower, in the remainder of this paper, we will only analyze those of the time-frequency descriptors.

Also in the case of the linear spectrogram, the obtained correlations are comparable in terms of magnitude order to the ones 


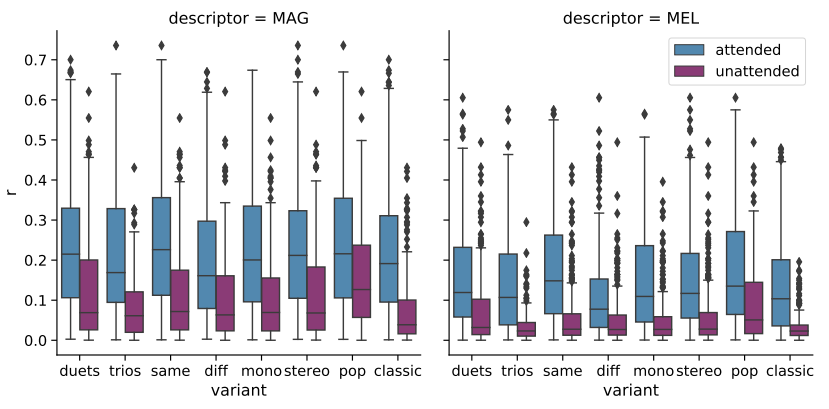

Figure 3: $r_{\text {attended }}$ and $r_{\text {unattended }}$ scores for the all the stimuli variants. Only MAG and MEL descriptors are considered. $r_{\text {attended }}$ and $r_{\text {unattended }}$ distributions are significantly different for all the variants ( $p<0.001$, non-parametric Wilcoxon test).

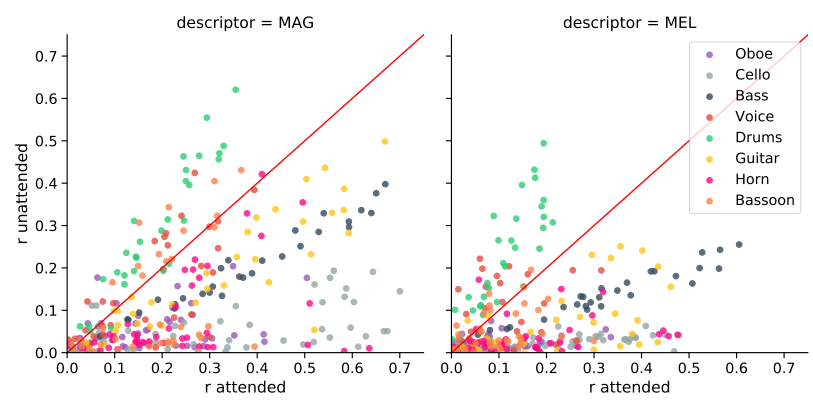

Figure 4: $r_{\text {attended }}$ is plotted against $r_{\text {unattended }}$ for each duet in the test set (only MAG and MEL descriptors). Data points below the red line $r_{\text {attended }}=r_{\text {unattended }}$, are classified correctly. Data points in the bottom-right corner are classified correctly with a large margin. The instruments are marked with different colors.

obtained previously by [6] for speech in a different setting. Even if MAG correlations with the attended instrument (median $r=0.215$ ) are in general higher than the MEL ones (median $r=0.119$ ), the gap between $r_{\text {attended }}$ and $r_{\text {unattended }}$ is higher for MEL as can be seen in Figure 2, leading to better decoding performances. Also in this case, many misclassifications happen when the reconstructed stimulus quality is low, i.e. when both $r_{\text {attended }}$ and $r_{\text {unattended }}$ coefficients are very low $(r<0.2)$ and close (see Figure 4$)$. Here the model is accounting for effects which are probably more related to the whole mixtures than individual instruments. When $r_{\text {attended }}$ is high, usually the corresponding $r_{\text {unattended }}$ is low, meaning that the model is discriminating the two instruments.

As expected, the number of instruments in the mixtures seems to affect the performances, which are better for duets than trios. This is reasonable, as the more sources we have, the more difficult is the attention decoding task. The spatial rendering, i.e. whether the stimuli are played in a mono or a stereo setting, does not significantly influence the decoding performance and the correlations values, though surprisingly, mono playback seems to be more advantageous than stereo in terms of F1 scores, with the differences not being statistically significant $(p>0.05$, non-parametric Wilcoxon test). It seems that the subjects are not helped by a different spatial localization of the instruments to attend to one of them or, at least, the EEG is not linearly tracking any of the related information.

The genre, instead, is highly influencing the performances. Both the time-frequency descriptors behave much better for the
Classical music mixtures compared to Pop ones as can be seen in Table 1. This probably happens because the nature of the Pop excerpts used as stimuli is mostly repetitive musical patterns, which are essentially rhythmical. In our dataset, this is particularly true in mixtures with the Drums and the Bass, which usually have to guide the rhythm and tempo. The Classical mixtures used are inherently different: they exhibit long melodic lines which can be translated in well-defined varying pitch contours. Thus, the very good performances on the classical pieces can be explained by the fact that our model is tracking well the pitch/harmonic contour of the attended instrument. Usually, when one attends to an instrument one focuses on following the melody line or rhythm played.

That is why we tested if our models are invariant to the melody/rhythmical pattern that is played. In fact, the performance clearly changes when we test the models on different musical pieces from those which were used for training, and is better when the melody/rhythmical pattern remains the same. It is worth clarifying that even in this case, though the same solo excerpt is used during training and testing, during the latter, that solo excerpt is played as part of a mixture (duet or trio) and the EEG response is obviously completely different from that of the training with the soloonly stimulus. This performance degradation observed when the pitch contours varies between training and testing is coherent with the explanation we gave before for the difference of performance among the genres. However, this also means that the generalization ability of the considered models is limited. Even if the models are not invariant to the changing pitch contour, the performance still remains significantly better than chance for the two time-frequency representations. In this case, the linear spectrogram seems to be more robust than the Mel one. The lower performance on the Pop excerpts can be explained also by the fact that the Drums are always misclassified as Bass. Our tentative explanation is that when the subject is listening to the Drums and the Bass, the brain's activity is mostly tracking the rhythm. More experiments using recordings with clearer distinction between melody and rhythm will be needed to confirm these initial findings.

\section{CONCLUSION}

We have shown that EEG-based auditory attention decoding based on a simple linear regression model yields promising results for decoding the attended instrument in polyphonic music. Through experimental evaluation we have shown that the EEG tracks musically-relevant features which are highly correlated with the time-frequency representation of the attended source and only weakly correlated with the unattended one. Moreover, we have shown that we are actually tracking attention since these features are related with the attended source and not with the mixture in its whole. However, it seems that the models are mostly tracking the pitch contour that is played by the instrument, which reduces its generalization capabilities.

The main limitation is that the proposed paradigm employs the separate "clean" sources of each instrument present in the mixture (to correlate their feature representation to the one predicted with the EEG data). This condition is never met in realistic scenarios where only the mixtures are available. Moreover, our linear model is not tracking all the non-linearity of the EEG signals. Future work will look into testing the proposed approach with non linear models and without access to the clean sources. Moreover, we will extend this approach to a music instrument classification problem, where we assume the target instrument is not known in advance. 


\section{REFERENCES}

[1] N. Ding and J. Z. Simon, "Emergence of neural encoding of auditory objects while listening to competing speakers," Proceedings of the National Academy of Sciences, vol. 109, no. 29, pp. $11854-11859,2012$.

[2] S. Akram, J. Z. Simon, S. A. Shamma, and B. Babadi, "A state-space model for decoding auditory attentional modulation from meg in a competing-speaker environment," in $\mathrm{Ad}$ vances in Neural Information Processing Systems, 2014, pp. 460-468.

[3] C. Brodbeck, A. Presacco, and J. Z. Simon, "Neural source dynamics of brain responses to continuous stimuli: speech processing from acoustics to comprehension," NeuroImage, vol. 172, pp. 162-174, 2018.

[4] N. Mesgarani, S. V. David, J. B. Fritz, and S. A. Shamma, "Influence of context and behavior on stimulus reconstruction from neural activity in primary auditory cortex," Journal of neurophysiology, 2009.

[5] B. N. Pasley, S. V. David, N. Mesgarani, A. Flinker, S. A. Shamma, N. E. Crone, R. T. Knight, and E. F. Chang, "Reconstructing speech from human auditory cortex," PLoS biology, vol. 10, no. 1, p. e1001251, 2012.

[6] N. Mesgarani and E. F. Chang, "Selective cortical representation of attended speaker in multi-talker speech perception," Nature, vol. 485, no. 7397, p. 233, 2012.

[7] J. A. O'sullivan, A. J. Power, N. Mesgarani, S. Rajaram, J. J. Foxe, B. G. Shinn-Cunningham, M. Slaney, S. A. Shamma, and E. C. Lalor, "Attentional selection in a cocktail party environment can be decoded from single-trial eeg," Cerebral Cortex, vol. 25, no. 7, pp. 1697-1706, 2014.

[8] M. J. Crosse, G. M. Di Liberto, A. Bednar, and E. C. Lalor, "The multivariate temporal response function (mtrf) toolbox: a matlab toolbox for relating neural signals to continuous stimuli," Frontiers in human neuroscience, vol. 10, p. 604, 2016.

[9] K. L. Hyde, I. Peretz, and R. J. Zatorre, "Evidence for the role of the right auditory cortex in fine pitch resolution," Neuropsychologia, vol. 46, no. 2, pp. 632-639, 2008.

[10] S. Kumar, W. Sedley, K. V. Nourski, H. Kawasaki, H. Oya, R. D. Patterson, M. A. Howard III, K. J. Friston, and T. D. Griffiths, "Predictive coding and pitch processing in the auditory cortex," Journal of Cognitive Neuroscience, vol. 23, no. 10, pp. 3084-3094, 2011.

[11] Y. Nan and A. D. Friederici, "Differential roles of right temporal cortex and broca's area in pitch processing: evidence from music and mandarin," Human brain mapping, vol. 34, no. 9, pp. 2045-2054, 2013.

[12] C. J. Plack, D. Barker, and D. A. Hall, "Pitch coding and pitch processing in the human brain," Hearing Research, vol. 307, pp. 53-64, 2014.

[13] A. Caclin, M.-H. Giard, B. K. Smith, and S. McAdams, "Interactive processing of timbre dimensions: A garner interference study," Brain research, vol. 1138, pp. 159-170, 2007.

[14] S. Deike, B. Gaschler-Markefski, A. Brechmann, and H. Scheich, "Auditory stream segregation relying on timbre involves left auditory cortex," Neuroreport, vol. 15, no. 9, pp. 15111514, 2004.
[15] K. N. Goydke, E. Altenmüller, J. Möller, and T. F. Münte, "Changes in emotional tone and instrumental timbre are reflected by the mismatch negativity," Cognitive Brain Research, vol. 21, no. 3, pp. 351-359, 2004.

[16] I. Sturm, "Analyzing the perception of natural music with eeg and ecog," Ph.D. thesis, 2016.

[17] F. Cong, A. H. Phan, Q. Zhao, A. K. Nandi, V. Alluri, P. Toiviainen, H. Poikonen, M. Huotilainen, A. Cichocki, and T. Ristaniemi, "Analysis of ongoing eeg elicited by natural music stimuli using nonnegative tensor factorization," in EUSIPCO. IEEE, 2012, pp. 494-498.

[18] I. Sturm, M. Treder, D. Miklody, H. Purwins, S. Dähne, B. Blankertz, and G. Curio, "Extracting the neural representation of tone onsets for separate voices of ensemble music using multivariate eeg analysis." Psychomusicology: Music, Mind, and Brain, vol. 25, no. 4, p. 366, 2015.

[19] S. Stober, T. Prätzlich, and M. Müller, "Brain beats: Tempo extraction from eeg data." in ISMIR, 2016, pp. 276-282.

[20] M. H. Thaut, "Rhythm, human temporality, and brain function," Musical communication, pp. 171-191, 2005.

[21] A. Ofner and S. Stober, "Shared generative representation of auditory concepts and eeg to reconstruct perceived and imagined music." in ISMIR, 2018, pp. 392-399.

[22] I. Sturm, S. Dähne, B. Blankertz, and G. Curio, "Multi-variate eeg analysis as a novel tool to examine brain responses to naturalistic music stimuli," PloS one, vol. 10, no. 10, p. e0141281, 2015.

[23] R. S. Schaefer, P. Desain, and J. Farquhar, "Shared processing of perception and imagery of music in decomposed eeg," Neuroimage, vol. 70, pp. 317-326, 2013.

[24] M. S. Treder, H. Purwins, D. Miklody, I. Sturm, and B. Blankertz, "Decoding auditory attention to instruments in polyphonic music using single-trial eeg classification," Journal of neural engineering, vol. 11, no. 2, p. 026009, 2014.

[25] B. Kaneshiro, D. T. Nguyen, J. P. Dmochowski, A. M. Norcia, and J. Berger, "Naturalistic music eeg dataset - hindi (nmedh)," https://purl.stanford.edu/sd922db3535, 2016.

[26] S. Losorelli, D. T. Nguyen, J. P. Dmochowski, and B. Kaneshiro, "Nmed-t: A tempo-focused dataset of cortical and behavioral responses to naturalistic music," 2017.

[27] S. Stober, A. Sternin, A. M. Owen, and J. A. Grahn, "Towards music imagery information retrieval: Introducing the openmiir dataset of eeg recordings from music perception and imagination." in ISMIR, 2015, pp. 763-769.

[28] H. Akbari, B. Khalighinejad, J. L. Herrero, A. D. Mehta, and N. Mesgarani, "Towards reconstructing intelligible speech from the human auditory cortex," Scientific reports, vol. 9, no. 1, p. 874, 2019.

[29] B. Blankertz, S. Lemm, M. Treder, S. Haufe, and K.-R. Müller, "Single-trial analysis and classification of erp componentsa tutorial," NeuroImage, vol. 56, no. 2, pp. 814-825, 2011.

[30] E. W. Noreen, Computer-intensive methods for testing hypotheses. Wiley New York, 1989.

[31] A. Yeh, "More accurate tests for the statistical significance of result differences," in Proceedings of the 18th conference on Computational linguistics-Volume 2. Association for Computational Linguistics, 2000, pp. 947-953. 